\title{
On Citations, Uses, and Informed Guesswork: A Response to Line
}

\section{Robert N. Broadus}

The use of the ISI Journal Citation Reports (JCR) to identify little-cited journals as candidates for cancellation remains, despite Line's comments to the contrary, an inexpensive means to approach a problem that faces most academic libraries. While the approach can only serve as a rough guide, convenience of use and low cost make citation analysis an appropriate technique with which to search for periodical titles to cancel. When applied with other situational and pragmatic considerations, the use of JCR can make an otherwise burdensome process far easier to conduct.

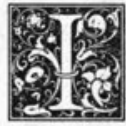

am pleased to continue this discussion with Maurice Line, whose writings are well known and for whom I have great respect.

I take it that the "crucial practical issue" that he says I have missed is that "librarians want to know . . . what additional titles to buy." I agree that the problem is important, and that eventually the library will produce one list representing decisions about both additions and deletions, but see no reason why the stages cannot be separated at some point in order to take advantage of useful evidence. The scope of my paper is limited to cancellations. If the library proposes to add titles, it may have to consider for deselection more than the hypothetical 20 percent of its present list.

In short, my paper recommends identifying a list of little-cited journals as candidates for possible deselection by use of the Journal Citation Reports (JCR) because this procedure would be relatively inexpensive and yet likely to prove nearly as good as a more costly use study-a kind of study more feasible if the entire collection is to be evaluated. I did not suggest ordering the little-cited journals according to rank.

Line's chain of thought seems to be: (1) journal ranks by use are unstable; (2) ranks by citation counts are stable; (3) therefore, citations do not reflect actual use; and (4) therefore, since both use and citation studies are very imperfect predictors of local needs or wants, we may as well have the users say what they want and let them take the consequences.

We could compare our recommended procedures more satisfactorily had Line given more details. It is not clear whose opinions would be sought or how they would be tabulated and then acted upon. Since both selection and deselection decisions are to be made at exactly the same time, will each voter be given a list of all possible titles (with prices) and asked to pick so many dollars' or pounds' worth? With thousands of titles to be considered, this task will be time-consuming, both for the users and for the librarians trying to make sense of the votes. Will runoff elections be required-several, perhaps, before the final list can be formed? There are

Robert N. Broadus is professor in the School of Library Science at the University of North Carolina, Chapel Hill, North Carolina 27514. 
more difficulties with Line's proposal than "declining local interests.'

Note also the fact that I recommend that users (as faculty) be asked to review the list of potential losers. Giving them this shorter list would produce more manageable data in less time. I don't see that users' judgments would be damaged by their knowing that these journals were seldom cited.

Not entirely satisfied with his solution, Line suggested monitoring the use of fringe periodicals. He is right about such a practice being "tedious and perhaps expensive," especially if the study were thorough and most of the monitored use were in-house. A few lapses in the monitoring of fringe titles would seriously distort the results. Nor would the procedure deal with the "crucial practical issue" of what titles ought to be added. If use figures are as unstable as he implies, would even three years' data be sufficient? I do agree that it would be interesting to see whether the group that has not been cited is substantially the same as the group that is not used.

Line's other alternative, "informed guesswork," seems indefinite also. What- ever the faults of methods currently available for solving the problem, we ought to be trying to find better ones than this.

I really should have mentioned the fact that highly cited periodicals may be candidates for cancellation, but that involves a good deal of discussion. There are, of course, other obvious reasons for dropping certain subscriptions, e.g., discontinuance of a program, school, or department. Under ordinary circumstances, though, I would hesitate to cancel a highly cited journal, circumstances may change and the journal would again become necessary for programatic support. I certainly would hate to drop such a title on the basis of a close vote, knowing the possibility of a subsequent reversal, leaving a gap of two or three years in the run.

Marginal titles present terrible dilemmas when a subscription list is made up. In my paper I tried to outline this difficulty, and how the $J C R$ can provide relatively low-cost information, as a place to start. I did not "admit" that they are a rough guide; I stressed it. But in a situation as complex as this one, we need all the evidence we can get. The bath water may be dirty, but let's hold on to the baby. 